\title{
Erratum to: Is the evaluation of millennial changes in stature reliable? A study in southern Europe from the Neolithic to the Middle Ages
}

Patrizia Martella $^{1} \cdot$ Maurizio Brizzi $^{2} \cdot$ Emanuele Sanna $^{1}$

Published online: 30 September 2016

(C) Springer-Verlag Berlin Heidelberg 2016

Erratum to: Archaeol Anthropol Sci

DOI 10.1007/s12520-016-0374-4

Table 1 in the original version of this article contains some incorrect dates. The complete, corrected table is as follow:

The online version of the original article can be found at doi:10.1007 /s12520-016-0374-4.

Patrizia Martella

martellapatrizia@gmail.com

1 Department of Environmental and Life Science, Section of Neuroscience and Anthropological Science, Cittadella Universitaria, University of Cagliari, 09042 Monserrato, Italy

2 Department of Statistical Sciences "Paolo Fortunati", University of Bologna, Via Belle Arti, 41 Bologna, Italy 
Table 1 Sardinian skeletal material: archeological sites, dating and provenance of samples
Archaeological site

Dating or Period (reference)

Provenance of samples

San Benedetto-Iglesias

Is Aruttas-Oristano

Capo Pecora-Arbus

Tueri Cave-Perdasdefogu

Lu Maccioni Cave-Alghero

Li Muri-Arzachena

Ingurtosu Mannu-Donori

Sa Serra Masi-Siliqua

Mitza Salida-Masullas

Genna Cuccureddu-Baunei

San Saturnino Church, Cagliari $8^{\text {th }}$ $-10^{\text {th }}$ century CE.

San Michele Church, Bono (Sassari) $16^{\text {th }}-19^{\text {th }}$ century CE
Late Neolithic 3978-3648 cal BC $2 \sigma$ (Lai 2009)

Nuragic 1433-1130 cal BC $2 \sigma$ (Lai 2009)

Nuragic $1261 \pm 105$ cal. BC $1 \sigma$ (Sanna 2006)

Nuragic $2880 \pm 60$ BP no cal.

$$
\text { (Sanna 2006) }
$$

Nuragic $2800 \pm 60$ BP no cal.

$$
\text { (Sanna 2006) }
$$

Nuragic (Germanà 1995)

Nuragic $1205-910$ cal BC $2 \sigma$ (Martella et al. 2014)

Nuragic 1690-1400 cal BC $2 \sigma$ (Martella et al. 2014)

Imperial Roman (Manos and Floris 2005)

Roman Period (personal communication R. Floris

Early Medieval Period (Floris and Usai 1997)

Modern Age (Maxia and Fenu, 1963)
MSAE $^{1}$

SASAR $^{2}$

MSAE

MSAE

MSAE

MSAE

MSAE

SASAR

SASAR

SASAR

SASAR

MSAE

${ }^{1}$ MSAE: Sardinian Museum of Anthropology and Ethnography

${ }^{2}$ SASAR: Archaeological Superintendence of Sardinia 